\title{
Valores normativos do comportamento sedentário em idosos
}

\section{Normative values of sedentary behavior in the elderly}

\author{
Lucas Lima Galvão ${ }^{1}$ \\ Sheilla Tribess ${ }^{2}$ \\ Joilson Meneguci ${ }^{2}$ \\ Emille Camila de Oliveira Santos ${ }^{1}$ \\ Rafaela Gomes dos Santos ${ }^{2}$ \\ Valfredo Ribeiro Dórea ${ }^{1}$ \\ Jair Sindra Virtuoso Júnior ${ }^{2}$ \\ Douglas de Assis Teles Santos ${ }^{1, *}$
}

\section{Resumo}

Objetivo: identificar os valores normativos do CS em idosos do Estudo ELSIA. Estudo observacional, do tipo analítico com delineamento transversal utilizando método exploratório survey. Métodos: A amostra foi composta por 473 sujeitos de ambos os sexos com idade igual ou superior a 60 anos, agrupados por sexo e idade em três grupos, 60-69 anos, 70-79 anos e 80 anos ou mais, moradores da cidade de Alcobaça, Bahia. O comportamento sedentário foi determinado através da seção tempo sentado (minutos por dia) do Questionário Internacional de Atividade Física. Os procedimentos da estatística descritiva foram utilizados para identificar a amostra com a distribuição da frequência, percentil (P10, P20, P30, P40, P50, P60, P70, P80, P90), cálculo de medida de tendência central (média e mediana) e de dispersão (amplitude de variação, desvio-padrão) e estatística inferencial (teste Kruskal-Wallis), $\mathrm{p}<0,05$. Resultados: O tempo exposto ao comportamento sedentário, de acordo com a faixa etária, respectivamente para homens e mulheres variaram de 189,2 e 210,2min/dia a 648,5 e 606,2 min/dia na faixa etária de 60-69 anos, 222,0 e 283,5 e 246,6 min/dia a 660,5 e 680,0 min/dia na faixa etária de 7079 anos e de 280,0 e 204,3 min/dia a 671,4 e $778,8 \mathrm{~min} /$ dia para a faixa etária de 80 anos ou mais. Conclusão: O presente estudo possibilitou identificar os valores normativos do comportamento sedentário entre os idosos de Alcobaça, BA, representando uma detalhada distribuição da população e de seu CS demonstrando que quanto mais elevada à idade, maior o tempo exposto ao CS, servindo ainda como parâmetro de avaliação e mudança de comportamento ao aumento do nível de prática de atividade física.
\end{abstract}

Palavras-chave: idoso, saúde, estilo de vida sedentário.

\begin{abstract}
Objective: to identify the normative values of CS in the elderly of the ELSIA Study. Methods: An observational study of the analytical type with a cross-sectional design using an exploratory survey method. The sample consisted of 473 subjects of both sexes aged 60 and over, grouped by sex and age in three groups, 60-69 years, 70-79 yearsand 80 years or more, residents of the city of Alcobaça, Bahia. Sedentary behavior was determined through the sitting time (minutes per day) section of the International Physical Activity Questionnaire. Descriptive statistics procedures were used to identify the sample with the frequency distribution, percentile (P10, P20, P30, P40, P50, P60, P70, P80, P90), calculation of central tendency measure (mean and median) and (range of variation, standard deviation) and inferential statistics (Kruskal-Wallis test), $\mathrm{p}<0.05$. Results: The time exposed to sedentary behavior, according to the age range, respectively for men and women ranged from 189.2 and $210.2 \mathrm{~min} /$ day to 648.5 and $606.2 \mathrm{~min} /$ day in the age range of $60-69$ years, 222.0 and 283.5 and $246.6 \mathrm{~min} /$ day at 660.5 and $680.0 \mathrm{~min} /$ day in the age group of $70-79$ and 280.0 and $204.3 \mathrm{~min} /$ day at 671.4 and $778.8 \mathrm{~min} / \mathrm{day}$ for the age group of 80 years or more. Conclusion: The present study made it possible to identify the normative values of sedentary behavior among the elderly of Alcobaça, Bahia, representing a detailed distribution of the population and their SB, showing that thehigher the age, thelonger the time exposed to $\mathrm{SB}$, and also serves as an evaluation parameter and behavior change to the increased level of physical activity practice.
\end{abstract}

Keywords: aged, health, sedentary lifestyle
Afiliação dos autores

${ }^{1}$ Universidade do Estado da Bahia, Teixeira de Freitas, Bahia, Brasil. ${ }^{2}$ Universidade Federal do Triângulo Mineiro, Uberaba, Minas Gerais, Brasil.

${ }^{\star}$ Autor correspondente

Colegiado de Educação Física, Universidade do Estado da Bahia Avenida Kaikan s/n, Setor universitário, CEP: 45992-255 Teixeira de Freitas, Bahia, Brasil e-mail: datsantos@uneb.br

Conflito de interesses

Os autores declararam não haver conflito de interesses.

Processo de arbitragem 


\section{Introdução}

As mudanças no perfil demográfico vêm sendo intensamente investigadas em estudos populacionais, os resultados encontrados sugerem cada vez mais planejamentos e reformulações de políticas sociais, econômicas e de saúde ${ }^{1}$. O Brasil vem passando por transformações expressivas em sua composição etária, apresentando um acelerado processo de envelhecimento com diversas alterações relevantes no quadro de morbi-mortalidade ${ }^{2}$.

O país ainda vive um declínio nos níveis de mortalidade e fecundidade, provocando alterações em sua estrutura etária ${ }^{1}$. O envelhecimento é entendido como uma fase comum na vida do ser humano, causa diversas alteraçõesmorfológicas e fisiológicas, sendo gradual, universal e irreversível, provocando perda funcional progressiva no organismo ${ }^{3}$.

O segmento populacional de maior aumento na população brasileira são os idosos, passando de 14,2 milhões em 2000, para 19,6 milhões em 2010, devendo atingir 41,5 milhões em 2030 e 64 milhões em 2050, podendo representar 226 idosos ou mais para cada 100 crianças ou adolescentes ${ }^{1,4}$. A população idosa está mais suscetível ao acometimento de doenças crônicas não transmissíveis (DCNT's). Nos últimos anos as DCNT são as responsáveis por grande número de mortes, cerca de $70 \%$, constituindo um grande problema de saúde podendo provocar a perda de qualidade de vida, gerando incapacidade e alto grau de limitação em suas atividades de trabalho e lazer ${ }^{5,6}$.

Diversas pesquisas têm demonstrado associação das DCNT a fatores de riscos como tabagismo, etilismo, excesso de peso e elevado comportamento sedentário, podendo agravar seu estado de saúde ${ }^{3,7}$. Este tempo gasto em comportamento sedentário (CS) está fortemente relacionado ao aumento do risco de contrair DCNT e comprometer a qualidade de vida ${ }^{7,8}$.

O CS é representado por atividades de pequena movimentação do músculo esquelético, com gasto energético s 1,5 MET, incluindo atividades como uso do telefone, assistir televisão, usar computador ou videogame ${ }^{2,9}$, atividades de tela discutida por Tremblay et al. ${ }^{10}$ como tempo de tela sedentário, caracterizado pela utilização destes dispositivos em CS, como conversar com amigos, todas realizadas na posição sentada ou deitada $^{11-13}$

Evidências demonstram a tendência dos idosos apresentarem CS mais elevado comparado a outros grupos etários $^{9,14}$, como observado no estudo de Lopes et al.15 no qual as idosas se referiram sem vontade de realizar atividades com outras pessoas ou se diziam indispostas a praticar atividade física por já trabalharem o suficiente.

Santos et al. ${ }^{2}$, apresentaram em revisão sistemática, um aumento crescente de estudos em torno do CS em pessoas idosas, no entanto, mesmo com esse aumento, ainda há lacunas acerca dessa área de investigação. Não existe um consenso entre os pesquisadores da área, quanto ao melhor ponto de corte que discrimine a duração do tempo sentado na predição de riscos à saúde do idoso ${ }^{2,11,13}$, entretanto os resultados a partir de quatro horas de exposição ao CS parece ser consistente ${ }^{2,9}$.

0 rápido envelhecimento da população tem profundas implicações, trazendo importantes desafios para a sociedade, fazendo necessário avançar ainda mais sobre os estudos e sua relação com o CS. Visto a escassez destes e de sua relevância para auxiliar no planejamento, avaliação e prescrição de programas de promoção da saúde, o desenvolvimento de valores normativos, torna-se relevante e possível ferramenta efetiva em políticas públicas de saúde. Assim, o objetivo desta pesquisa é predizer os valores normativos do CS em idosos.

\section{Métodos}

Estudo observacional, do tipo analítico com delineamento transversal utilizando métodos exploratórios surveys, realizado no município de Alcobaça, localizada na região do Extremo Sul, do Estado da Bahia.

O presente estudo faz parte do Estudo Longitudinal de Saúde do Idoso de Alcobaça - BA (ELSIA), projeto de pesquisa foi desenvolvido em conjunto entre a Universidade do Estado da Bahia (UNEB) e a Universidade Federal do Triângulo Mineiro (UFTM). Estudo aprovado pelo Comitê de Ética em Pesquisa da UFTM sob o parecer número 966.983.

A amostra deste estudo foi composta por 473 pessoas de ambos os sexos com idade igual ou superior a 60 anos. As pessoas selecionadas não deviam conter comprometimento cognitivo grave no Mini Exame do Estado Mental (MEEM), desenvolvido originalmente por Folstein et al. ${ }^{16}$ e adaptado para população brasileira Almeida ${ }^{17}$; não possuir dificuldade grave na acuidade visual e auditiva, não fazer uso de cadeiras de rodas, não possuir sequelas graves de acidente vascular encefálico (AVE) com perda localizada de força e não possuir doença em estágio terminal.

Para a visita domiciliar os pesquisadores utilizaram como referência dados disponibilizados pela Secretaria Municipal de Saúde de Alcobaça. O contato foi realizado com os idosos por meio de visita domiciliar, informando dos objetivos e solicitando a participação deles na pesquisa, ocorrendo de forma voluntaria. Depois de aceito foi entregue um termo de consentimento livre e esclarecido (TCLE) e submetidos a um questionário, aplicado em forma de entrevista, por acadêmicos e profissionais de Educação Física, devidamente treinados. A coleta de dados teve duração de julho a setembro de 2015.

O roteiro de entrevista foi previamente testado em estudo piloto (para identificação dos índices psicométricos), sendo construído mediante a composição de outros instrumentos abordando características sociodemográficas e indicadores de saúde (enfermidades, sintomatologia depressiva) ${ }^{18}$, atividade física ${ }^{19}$, transtornos cognitivos leves ${ }^{16}$, incapacidade funcional nas atividades da vida diária (AVD) ${ }^{20}$ e instrumentais (AIVD) ${ }^{21}$ e risco de desnutrição ${ }^{22}$.

O CS foi determinado pelo tempo gasto sentado, avaliado a partir das questões de tempo sentado em um dia habitual da semana e um dia habitual de fim de semana, de acordo com o Questionário Internacional de Atividade Física (IPAQ) ${ }^{23}$, validado para a população idosa brasileira ${ }^{19,24}$. O tempo gasto sentado total, minutos/dia, foi determinado a partir da média ponderada do tempo sentado em um dia de semana e um dia de final de semana: [(tempo sentado em um dia de semana $\times 5+$ tempo sentado em um dia de final de semana $x$ 2) / 7].

Para confecção do banco de dados foi utilizado o software Epidata, versão $3.1 \mathrm{~b}$, e as análises por meio do software estatístico SPSS 20.0 (Statistical Package for the Social Sciences). A normalidade dos dados foi verificada pelo teste Kolmogorov-Smirnov e o teste Kruskal-Wallis foi utilizado para comparar as características dos idosos entre os grupos das faixas etárias, adotando $\mathrm{p}<0,05$. Foi utilizada estatística descritiva dos percentis P10, P20, P30, P40, P50, P60, P70, $\mathrm{P} 80, \mathrm{P} 90$ para ambos os sexos e idades.

\section{Resultados}

A população estudada foi constituída por 473 sujeitos, sendo 297 mulheres $(62,8 \%)$ e 176 homens $(37,2 \%)$, com idade entre 60 e 97 anos, média etária de 70,25 \pm 8,25 anos, sendo a média para as mulheres $69,89 \pm 8,16$ anos e para os homens $70,85 \pm$ 8,39 anos e constituindo em sua maioria $(55,2 \%)$ a faixa etária de 60 a 69 anos.

As características antropométricas dos idosos são descritas na Tabela 1.

Tabela 1

Características dos participantes.

\begin{tabular}{lcccc}
\hline & Total $(\mathrm{n}$ & $60-69$ & $70-79(\mathrm{n}=$ & $\geq 80(\mathrm{n}=72)$ \\
& $=473)$ & $(\mathrm{n}=261)$ & $140)$ & \\
\hline Massa corporal & $67,86 \pm$ & $69,71 \mathrm{a} \pm$ & $68,16 \mathrm{~d} \pm$ & $60,61 \mathrm{a}, \mathrm{d} \pm$ \\
$(\mathrm{kg})$ & 14,13 & 14,44 & 13,13 & 12,66 \\
Estatura $(\mathrm{cm})$ & $157,73 \pm$ & $158,13 \mathrm{~b} \pm$ & $158,75 \mathrm{e} \pm$ & $154,29 \mathrm{~b}, \mathrm{e} \pm$ \\
& 9,63 & 9,20 & 9,92 & 9,96 \\
IMC $(\mathrm{kg} / \mathrm{m} 2)$ & $27,30 \pm$ & $27,90 \mathrm{c} \pm$ & $27,10 \pm$ & $25,49 \mathrm{c} \pm$ \\
& 5,39 & 5,58 & 5,04 & 5,00 \\
\hline
\end{tabular}

$\mathrm{a}, \mathrm{dp}<0,001 ; \mathrm{bp}=0,006) ; \mathrm{cp}=0,007 ; \mathrm{ep}=0,004$.

Na tabela 2 são apresentados os valores dos percentis para a amostra geral (homens e mulheres) separado pelas faixas etárias. Os valores dos percentis da idade $\geq 80$ anos são superioresaos da 70-79 anos que por sua vez são superiores aos da 60-69 anos, exceto para o P10 onde o valor da faixa etária $70-79$ anos $(246,6)$ é superior ao da $\geq 80$ anos $(246,0)$.

A tabela 3 expressa os percentis para os idosos do sexo masculino estratificados pela faixa etária. Seguindo uma linearidade do P10 ao P90, onde os maiores valores sempre se encontram relacionados com idosos de mais idade. O primeiro grupo de 60-69 anos continham 89 sujeitos cujo percentis de P10 a P90 varia entre 
$189,2 \mathrm{~min} / \mathrm{dia}$ e $648,5 \mathrm{~min} / \mathrm{dia}$, o segundo grupo $70-79$ anos foi composto por 58 idosos com variação do P10 222,0 min/dia a P90 $660,5 \mathrm{~min} / \mathrm{dia}$. O último grupo composto por 29 homens com idades

Tabela 2

Valores Normativos do Comportamento Sedentário em Idosos em função da idade.

\begin{tabular}{|c|c|c|c|c|c|c|c|c|c|c|}
\hline \multicolumn{11}{|c|}{ Percentis Geral $(n=473)$} \\
\hline Faixa Etária & $\mathrm{n}$ & P10 & P20 & P30 & P40 & P50 & P60 & P70 & P80 & P90 \\
\hline $60-69$ anos & 261 & 206,8 & 278,2 & 327,5 & 368,4 & 408,7 & 445,1 & 481,4 & 540,0 & 623,7 \\
\hline $70-79$ anos & 140 & 246,6 & 319,8 & 350,9 & 381,7 & 414,2 & 483,7 & 535,3 & 601,1 & 670,1 \\
\hline$\geq 80$ anos & 72 & 246,0 & 335,5 & 395,0 & 422,8 & 456,4 & 524,0 & 600,0 & 642,0 & 744,0 \\
\hline
\end{tabular}

Valores dos percentis expressos em minutos por dia.

Tabela 3.

Valores Normativos do Comportamento Sedentário em Homens Idosos.

\begin{tabular}{|c|c|c|c|c|c|c|c|c|c|c|}
\hline \multicolumn{11}{|c|}{ Percentis homens $(n=176)$} \\
\hline Faixa Etária & $\mathrm{n}$ & P10 & $\mathrm{P} 20$ & P30 & $\mathrm{P} 40$ & P50 & P60 & P70 & P80 & P90 \\
\hline 60-69 anos & 89 & 189,2 & 271,4 & 340,0 & 365,7 & 406,4 & 445,7 & 487,1 & 580,0 & 648,5 \\
\hline 70-79 anos & 58 & 222,0 & 296,0 & 357,5 & 380,2 & 411,3 & 478,1 & 512,7 & 595,1 & 660,5 \\
\hline$\geq 80$ anos & 29 & 280,0 & 327,8 & 408,5 & 420,0 & 450,0 & 495,7 & 527,8 & 600,0 & 671,4 \\
\hline
\end{tabular}

$\mathrm{Na}$ tabela 4são apresentados os valores dos percentis para as mulheres idosas estratificado pelas três categorias de faixa etária. $\mathrm{Na}$ faixa etária de 60-69 anos fizeram parte 172 mulheres, enquanto que na faixa etária de 70-79 anos e 80 anos ou mais 82 e 43 mulheres respectivamente. A faixa etária de 80 anos ou mais de 80 ou mais apresentou P10 de 280,0 min/dia e P90 de 671,4 $\mathrm{min} /$ dia.

Tabela 4.

Valores Normativos do Comportamento Sedentário nas Mulheres Idosas por idade.

\begin{tabular}{|c|c|c|c|c|c|c|c|c|c|c|}
\hline \multicolumn{11}{|c|}{ Percentis mulheres $(n=297)$} \\
\hline Faixa Etária & $\mathrm{n}$ & $\mathrm{P} 10$ & P20 & P30 & $\mathrm{P} 40$ & P50 & $\mathrm{P} 60$ & P70 & P80 & P90 \\
\hline 60-69 anos & 172 & 210,2 & 280,4 & 316,2 & 368,8 & 412,8 & 444,2 & 477,2 & 527,2 & 606,2 \\
\hline 70-79 anos & 82 & 283,5 & 324,1 & 346,2 & 383,7 & 427,8 & 484,7 & 538,7 & 603,1 & 680,0 \\
\hline$\geq 80$ anos & 43 & 204,3 & 335,8 & 385,0 & 433,4 & 506,4 & 567,4 & 606,5 & 719,7 & 778,8 \\
\hline
\end{tabular}

Valores dos percentis expressos em minutos por dia.

\section{Discussão}

Comportamentos inadequados inclusive o tempo exposto em CS estão sendo constantemente citado em estudos como causador de diversos malefícios para a saúde da população, como, perda da funcionalidade, aumento do risco de AVC, câncer de cólon e câncer de mama, hipertensão arterial, depressão, diabetes tipo II, obesidade, doenças isquêmicas $2,5,12,14$

O baixo tempo de exposição ao CS e a prática regular de atividade física podem reduzir riscos de cardiopatia, AVC, câncer de cólon e mama, isso ocorre porque o exercício físico melhora o metabolismo da glicose, reduz o nível de gordura e diminui a pressão arterial ${ }^{12,25}$, além de aumento/manutenção da capacidade aeróbica, massa muscular, redução de mortalidade, prevenção de doenças coronárias, menor risco de fratura no quadril ou vertebral, melhora do perfil lipídico, redução da massa gorda e risco de sarcopenia, prevenção/controle de diabetes tipo II, redução da demência, melhora da autoestima e autoconfiança, diminuição da ansiedade e do estresse e melhora do humor e da qualidade de vida ${ }^{5,25}$

Bueno et al. $^{26}$ em estudo de revisão demonstrou quanto menor o nível de atividade física maiores seriam os gastos com o governo para com a saúde dos indivíduos, como exemplo gastos com medicação. Inversamente relacionado quanto mais ativo fisicamente menores os gastos do governo com medicamento de combate as doenças crônicas.

Segundo Haskellet al. ${ }^{27}$, as recomendações de Atividade física são de 150 minutos de atividade física de intensidade moderada semanais ou 75 minutos de atividade de intensidade vigorosa ou a combinação de ambas, além de exercícios de força ou flexibilidade ao menos duas vezes por semana. Diversos estudos demonstram que realizar ao menos 30 minutos apresenta os menores tempo exposto em comportamento sedentário (204,3 $\mathrm{min} / \mathrm{dia})$, com relação ao $60-69$ anos (210,2 $\mathrm{min} / \mathrm{dia})$ e $70-79$ anos (283,5 min/dia). A partir do P20 os valores seguem uma ordem crescente tanto para a idade quanto para os percentis. de atividade física vigorosa ou moderada (AFVM) reduz riscos de morbidades e mortalidades ${ }^{2,28}$, entretanto, evidências sugerem em indivíduos mesmo cumprindo estas recomendações, mas expostos à elevado tempo de CS, ainda encontram-se em risco à saúde ${ }^{2,11,12,28}$.

A Tabela 1 apresenta os valores normativos do CS em idosos em função da idade demonstrando quanto mais elevado à idade, maior o tempo exposto ao CS. O tempo médio de exposição ao CS foi de 408,7 min/dia (6,8 horas/dia), Santos ${ }^{14}$ em seu estudo encontrou valores maiores de tempo de CS, na cidade de Uberaba-MG, em relação aos encontrados neste estudo, sugerindo variáveis sociodemográficas influenciando em tal comportamento, dados superiores também foram encontrados em estudos recentes Bennie et al ${ }^{13}$ onde o tempo médio encontrado foi de $308 \mathrm{~min} /$ dia e de Silva et al. ${ }^{29}$ de 240 $\mathrm{min} / \mathrm{dia}$ de CS.

Este fato pode ocorrer pelo aumento de limitações físicas decorrentes da presença de doenças crônico-degenerativas, pelo receio de sair da rotina ou praticar atividade física, além de experiências vividas, outro fator determinante se enquadra na segregação social e superproteção dos filhos, favorecendo uma vida mais improdutiva e menos ativa sendo importante destacar que o desconhecimento das atividades pode contribuir para esta acomodação e superproteção ${ }^{15}$, novas investigações devem ocorrer para demonstrar se estes fatores são determinantes no aumento da exposição do CS.

As tabelas 2 e 3 apresentam os valores de CS dos idosos por sexo respectivamente homens e mulheres, os grupos apresentaram valores inferiores aos encontrados no estudo de Santos et al. ${ }^{14}$ que associou o percentil 75 (660,0 min/dia para homens e 633,7 min/dia para mulheres) de CS em seu estudo com presença de sintomas depressivos, indicando que idosos inativos fisicamente apresentam maior probabilidade de 
apresentar sintomas depressivos se comparados com idosos que são fisicamente ativos e gastam menos tempo em CS, mostrando ainda possível diminuição destes riscos mesmo com atividades físicas de intensidade leve.

Ao se observar os percentis de homens e mulher divididos, os valores das mulheres vão aumentando e ultrapassando dos homens em quase todos os grupos, aumentando o risco de perda de força de membros superiores, aptidão aeróbia e incapacidade funcional, estes já estão aliados ao processo de envelhecimento e se agravam com aumento do tempo exposto ao $\mathrm{CS}^{8,9,15}$.

Esse aumento do CS ligado à idade pode ser uma característica da velhice principalmente em países em desenvolvimento ou subdesenvolvidos, ligada a imagem do idoso como ser dependente e isolado ${ }^{8}$.

Não há na literatura ainda um ponto de corte discriminando quanto tempo exposto ao CS trariam agravos para a saúde.Entende-se que recomendações de atividade física apresentadas por Haskell et al. ${ }^{27^{3}}$ e quebras no CS são extremamente benéficas reduzindo riscos de sarcopenia, osteoporose, síndrome metabólica, diabetes, doenças coronárias e declínio cognitivo ${ }^{13,28,30}$. Silva et al. ${ }^{29}$ evidenciaram em interrupções no CS redução do HDL juntamente com os níveis de LDL, diminuindo colesterol total, revelando em idosos expostos a mais de 330,00 minutos/dia ao CS terem maior chance de desenvolver diabetes comparados a indivíduos expostos a tempo menor.Saleh et al. ${ }^{30}$ realizaram estudo de intervenção com intenção de diminuir o tempo de CS, demonstrando melhora do IMC, massa corporal, triglicérides e pressão arterial sistólica e diastólica.

O estudo aborda uma amostra representativa da população idosa do município possibilitando uma real visão do nível de CS dos moradores. Como limitações do estudo se deve o fato do questionário ser ferramenta de trabalho recordatório possibilitando um viés de esquecimento do entrevistado, no entanto, sendo comumente utilizado em estudos epidemiológicos pelo custo-efetividade.

\section{Conclusão}

O CS vem causando grande preocupação nos últimos anos, considerado fator de risco para a saúde. Os dados apresentam os percentis dos idosos residentes em Alcobaça, BA com idade igual ou superior a 60 anos, representando uma detalhada distribuição da população e de seu CS demonstrando quanto mais elevada à idade, maior o tempo exposto ao CS.

Os melhores comportamentos encontrados no estudo são de 206,9 min/dia (3,44 horas/dia) da faixa etária de 60-69 anos, sendo os piores no grupo de 80 anos ou mais de $744 \mathrm{~min} / \mathrm{dia}$ (12,4 horas) de CS, os homens apresentaram comportamentos melhores em relação as mulheres $189,3 \mathrm{~min} / \mathrm{dia}(3,15$ horas/dia) e 210,3 (3,50 horas/dia) respectivamente.

Este estudo possibilita o estabelecimento de valores normativos do CS entre os idosos. O desenvolvimento destes é relevante, especialmente pela escassez destes, possibilita a comparação e a avaliação desta população. Espera-se que essa informação sirva de subsídio para ampliar as políticas públicas de saúde, sendo possível estabelecer intervenções para redução do tempo exposto ao CS, aumentando o nível de atividade física.

\section{Referências}

1. IBGE. Indicadores Sociodemográficos e de Saúde no Brasil [Internet]. Rio de Janeiro: INSTITUTO BRASILEIRO GEOGRAFIA E ESTATÍSTICA; 2009 [cited 2017 May 1]. Available from: http://www.ibge.gov.br/english/estatistica/populacao/indic_sociosaude/200 9/indicsaude.pdf

2. Santos RG, Medeiros JC, Schmitt BD, Santos DAT, Damião R, Tribess S, et al. Comportamento Sedentário em Idosos: Uma Revisão Sistemática. Motricidade. 2015;11(3):171-86

3. Gottlieb MGV, Schwanke CHA, Gomes I, Cruz IBM. Envelhecimento e Longevidade no Rio Grande do Sul : um perfil histórico , étnico e de morbimortalidade dos idosos. Rev Bras Geriatr e Gerontol. 2006;14(2):365-80.
4. IBGE. Mudança demográfica no Brasil no início do século $X X \mid$ : subsídios para as projeções da população . Rio de Janeiro: Instituto Brasileiro de Geografia e Estatística; 2015. 156 p.

5. Maciel MG. Atividade física e funcionalidade do idoso. Motriz. 2010;16(4):1024-32

6. IBGE. Estimativa populacional 2014. Instituto Brasileiro de Geografia e Estatística. 2014.

7. González K, Fuentes J, Márquez JL. Physical Inactivity , Sedentary Behavior and Chronic Diseases. Korean J Fam Medice. 2017;38:111-5.

8. Pereira LC, Figueiredo M do LF, Beleza CMF, Andrade EMLR, Silva MJ na da, Pereira AFM. Fatores preditores para incapacidade funcional de idosos atendidos na atenção básica. Rev Bras Enferm. 2017;70(1):112-8.

9. Silva DAS, Mendonça BC de A, Oliveira ACC de. Qual é o impacto do comportamento sedentário na aptidão física de mulheres a partir de 50 anos de idade? Rev Bras Cineantropometria e Desempenho Hum. 2012;14(2):154-63.

10. Tremblay MS, Aubert S, Barnes JD, Saunders TJ, Carson V, LatimerCheung $A E$, et al. Sedentary Behavior Research Network (SBRN) Terminology Consensus Project process and outcome. Int $\mathrm{J}$ Behav Nutr Phys Act. 2017;14(75):1-17.

11. Owen N, Healy GN, Matthews CE, Dunstan DW. Too Much Sitting: The Population-Health Science of Sedentary Behavior. Exerc Sport Sci Rev. 2010;38(3):105-13

12. Meneguci J, Assis D, Santos T, Silva RB, Go- R, Sasaki JE, et al. Sedentary behavior: Concept, physiological implications and the assessment procedures. Motricidade. 2015;11(1):160-74.

13. Bennie JA, Pedisic Z, Van Uffelen JGZ, Gale J, Banting LK, Vergeer I, et al. The descriptive epidemiology of total physical activity, musclestrengthening exercises and sedentary behaviour among Australian adults - results from the National Nutrition and Physical Activity Survey. BMC Public Health. 2016;16(73):1-13.

14. Santos DAT, Virtuoso JS, Meneguci J, Sasaki JE, Tribess S. Combined Associations of Physical Activity and Sedentary Behavior With Depressive Symptoms in Older Adults. Issues Ment Health Nurs. 2017 Feb 10;38(3):15.

15. Lopes MA, Krug R de R, Bonetti A, Mazo GZ. Barreiras que influenciaram a não adoção de atividade física por longevas. Rev Bras Ciências do Esporte. 2016;38(1):76-83

16. Folstein MF, Folstein SE, McHugh PR. "MINI-MENTAL STATE" A practical method for grading the cognitive state of patients for the clinician. $J$ Psychiatr Res. 1975:12:189-98.

17. Almeida OP. Mine Exame do Estado Mental e o diagnóstico de demência no Brasil. Arq Neuropsiquiatr. 1998;56(3-B):605-12.

18. Almeida OP, Almeida SA. Confiabilidade da versão brasileira da escala de depressão em geriatria (GDS) versão reduzida. Arq Neuropsiquiatr. 1999;57(2-B):421-6

19. Benedetti TB, Mazo GZ, Barros MVG De. Aplicação do Questionário Internacional de Atividades Físicas para avaliação do nível de atividades físicas de mulheres idosas : validade concorrente e reprodutibilidade testereteste. Rev Bras Ciência e Mov. 2004;12(1):25-34.

20. Lino VTS, Pereira SRM, Camacho LAB, Ribeiro Filho ST, Buksman S. Adaptação transcultural da Escala de Independência em Atividades da Vida Diária ( Escala de Katz ). Cad Saúde Pública. 2008;24(1):103-12.

21. Santos RL dos, Virtuoso Júnior JS. Confiabilidade da versão brasileira da escala de atividades instrumentais da vida diária. Rev Bras em Promoção da saúde. 2008:21(1):290-6.

22. Guigoz $Y$, Vellas B. The Mini Nutritional Assessment (MNA) for grading the nutritional state of elderly patients: presentation of the MNA, history and validation. Nestle Nutr Workshop Ser Clin Perform Programme. 1999;1:3 11-2.

23. Rosenberg DE, Bull FC, Marshall AL, Sallis JF, Bauman AE. Assessment of Sedentary Behavior with the International Physical Activity Questionnaire. J Phys Act Heal. 2008;5(s1):S30-44.

24. Benedetti TRB, Antunes P de C, Rodriguez-Añez CR, Mazo GZ, Petrosk ÉL. Reprodutibilidade e validade do Questionário Internacional de Atividade Física (IPAQ) em homens idosos. Rev Bras Med do Esporte. 2007 Feb;13(1):11-6.

25. WHO. WHO | Physical Activity and Adults [Internet]. WHO. World Health Organization; 2015 [cited 2018 May 3]. Available from: http://www.who.int/dietphysicalactivity/factsheet adults/en/

26. Bueno DR, Marucci M de FN, Codogno JS, Roediger M de A. Os custos da inatividade física no mundo: estudo de revisão. Cien Saude Colet. 2016;21(4):1001-10.

27. Haskell WL, Lee I, Pate RR, Powell KE, Blair SN. Physical Activity and Public Health: Updated Recommendation for Adults From the American College of Sports Medicine and the American Heart Association. 2007;39:1423-34.

28. Katzmarzyk PT, Lee IM. Sedentary behaviour and life expectancy in the USA: A cause-deleted life table analysis. BMJ Open. 2012;2(4):1-8.

29. Silva R de C da, Meneguci J, Martins TI, Santos Á da S, Sasaki JE, Tribess $S$, et al. Association between time spent sitting and diabetes mellitus in older adults: a population-based study. Rev Bras Cineantropometria e Desempenho Hum. 2015;17(4):379-88.

30. Saleh ZT, Lennie TA, Mudd-Martin G, Bailey AL, Novak MJ, Biddle M, et al Decreasing sedentary behavior by 30 minutes per day reduces cardiovascular disease risk factors in rural Americans. Hear Lung J Acute Crit Care. 2015;44(5):382-6. 